\title{
Quantitative estimates in uniform and pointwise approximation by Bernstein-Durrmeyer-Choquet operators
}

\section{SORIN G. GAL and SORIN TRIFA}

\begin{abstract}
.
For the qualitative results of uniform and pointwise approximation obtained in [8], we present here general quantitative estimates in terms of the modulus of continuity and of a $K$-functional, in approximation by the generalized multivariate Bernstein-Durrmeyer operator $M_{n, \Gamma_{n, x}}$, written in terms of Choquet integrals with respect to a family of monotone and submodular set functions, $\Gamma_{n, x}$, on the standard $d$-dimensional simplex. If $d=1$ and the Choquet integrals are taken with respect to some concrete possibility measures, the estimate in terms of the modulus of continuity is detailed. Examples improving the estimates given by the classical operators also are presented.
\end{abstract}

\section{REFERENCES}

[1] Berdysheva, E. E., Uniform convergence of Bernstein-Durrmeyer operators with respect to arbitrary measure, J. Math. Anal. Appl., 394 (2012), 324-336

[2] Berdysheva, E. E., Bernstein-Durrmeyer operators with respect to arbitrary measure. II : Pointwise convergence, J. Math. Anal. Appl, 418 (2014), 734-752

[3] Berdysheva, E. E. and Jetter, K., Multivariate Bernstein-Durrmeyer operators with arbitrary weight functions, J. Approx. Theory, 162 (2010), 576-598

[4] Choquet, G., Theory of capacities, Ann. Inst. Fourier (Grenoble), 5 (1954), 131-295

[5] Denneberg, D., Non-Additive Measure and Integral, Kluwer Academic Publisher, Dordrecht, 1994

[6] Dubois, D. and Prade, H., Possibility Theory, Plenum Press, New York, 1988

[7] Gal, S. G., Approximation by Choquet integral operators, Ann. Mat. Pura Appl., online access, DOI : 10.1007/s10231-015-0495-x

[8] Gal, S. G. and Opris, B. D., Uniform and pointwise convergence of Bernstein-Durrmeyer operators with respect to monotone and submodular set functions, J. Math. Anal. Appl., 424 (2015), 1374-1379

[9] Gonska, H., Kacsó D. and Rasa, I., The genuine Bernstein-Durrmeyer operators revisited, Results Math., 62 (2012), 295-310

[10] Wang, Z. and Klir, G. J., Generalized Measure Theory, Springer, New York, 2009

Department of MATHEMATics ANd COMPUTER SCIENCE

UNIVERSITY OF ORADEA

UNIVERSITĂŢII 1, 410087 ORADEA, ROMANIA

E-mail address: galso@uoradea.ro

BABEŞ-BOLYAI UNIVERSITY

FACULTY OF MATHEMATICS AND INFORMATICS

M. KOGĂNICEANU 1, 400084 CLUj-NAPOCA, ROMANIA

E-mail address: sorin.trifa@yahoo.com

Received: 05.02.2016; In revised form: 26.03.2016; Accepted: 20.04.2016

2010 Mathematics Subject Classification. 41A36, 41A25, 28 A25.

Key words and phrases. Bernstein-Durrmeyer-Choquet operator, Choquet integral, modulus of continuity, $K$ functional.

Corresponding author: Sorin G. Gal; galso@uoradea.ro 